\title{
Post-surgical Extraoral Commissure Stent for Correction of Microstomia in a Child with Chemical Burns: A Clinical Report
}

\author{
${ }^{1}$ Nidhi Mangtani, ${ }^{2}$ Gunjan Pruthi, ${ }^{3}$ Veena Jain
}

\begin{abstract}
An 8-year-old male child presented with long-standing microstomia due to chemical burns six months before reporting to us. Challenges and limitations were difficult intraoral and extraoral impressions, partially erupted and carious teeth along with financial constraints and unavailability for frequent follow-ups as the patient hailed from a distant place with poor accessibility. Microstomia was treated surgically followed by delivery of removable acrylic extraoral commissure stent to prevent lip adhesions in the post-surgical phase. Fabrication technique of the appliance was innovative, and it involved a new technique for making an impression of the oral commissures. The appliance was effective, economical, easy to fabricate and required minimal follow-up visits and adjustments.
\end{abstract}

Keywords: Burns, Microstomia, Novel impression technique, Stent.

How to cite this article: Mangtani N, Pruthi G, Jain V. Post-surgical Extraoral Commissure Stent for Correction of Microstomia in a Child with Chemical Burns: A Clinical Report. J Postgrad Med Edu Res 2019;53(1):38-41.

Source of Support: Nil

Conflict of Interest: None

\section{BACKGROUND}

Accidental chemical or electric burns to the oral and perioral region in children result in scarring, with contraction of the tissues and constriction of the circumoral musculature, which if left untreated, subsequently lead to microstomia. ${ }^{1-3}$ Other consequences may include facial disfigurement, difficulty in feeding, psychological trauma to the patient and parents, abnormalities in speech, and difficulty in the maintenance of oral hygiene because of poor accessibility. ${ }^{4,5}$

Microstomia can be managed or prevented if the

\footnotetext{
${ }^{1}$ Reader, ${ }^{2}$ Assistant Professor, ${ }^{3}$ Professor

${ }^{1}$ Department of Prosthodontics, Maharishi Markandeshwar College of Dental Sciences and Research, Mullana, Ambala, Haryana, India

${ }^{2}$ Department of Prosthodontics, Oral Health Sciences Centre, Postgraduate Institute of Medical Education and Research, Chandigarh, India

${ }^{3}$ Department of Prosthodontics, Center for Dental Education and Research, All India Institute of Medical Sciences, New Delhi, India

Corresponding Author: Gunjan Pruthi, Assistant Professor, Department of Prosthodontics, Oral Health Sciences Centre, Postgraduate Institute of Medical Education and Research, Chandigarh. Email: gunjan_prostho@yahoo.co.in
}

patient is made to wear a commissural splint to maintain the commissures in their normal relationship during the healing process as it offers physical resistance to scar contracture. ${ }^{6}$ These splints/appliances can be either tooth or tissue borne; prefabricated or customized microstomia appliances which may be static or dynamic in function and are constructed to be placed intraorally or extraorally. The tissue-borne devices are mainly indicated in edentulous adult patients or children with partially erupted dentition. The tooth-borne appliances may be removable or fixed, promise better patient compliance and more acceptable appearance. ${ }^{7}$ Objectives of the appliance are to prevent scarring, minimize lip adhesions and contracture during healing and to obtain adequate mouth opening for the patient. ${ }^{8}$ Prerequisite is that appliance should be retentive and comfortable for the child to wear.

Factors influencing the choice of an appliance suitable for a particular patient include the type of injury, the age of the patient, condition, and status of dentition, compliance and manual dexterity, availability and financial status of the patient. ${ }^{9}$

Various appliances documented in literature have been used in patients with electric burns who reported within 7-10 days of the accident. ${ }^{8,10}$ Flexible or semi-flexible orthoses, similar to mouthguards have been described for patients with chemical burns. ${ }^{11,12}$ Intraoral appliances require impressions of maxillary and mandibular arches and a full complement of teeth to provide adequate retention to the appliance while extraoral appliances are bulky, may require intraoral anchorage, are difficult to fabricate and are unesthetic. ${ }^{710,13}$ Poor patient compliance, high cost, and requirement of frequent follow-up visits for adjustment of the appliance are few other limitations. Gorham ${ }^{14}$ has described the fabrication of a simple thermoplastic device that takes less than 10 minutes to make, requires no special equipment, and is very inexpensive. The thermoplastic material is contoured to spread the angles of the mouth and lips and is held in place by bite plates molded to the upper and lower teeth. But this requires a fully erupted dentition to provide retention to the appliance.

So, there was a need for a simple, easy to fabricate, an economical appliance for patients with a history of chemical burns, long-standing microstomia and partially erupted 

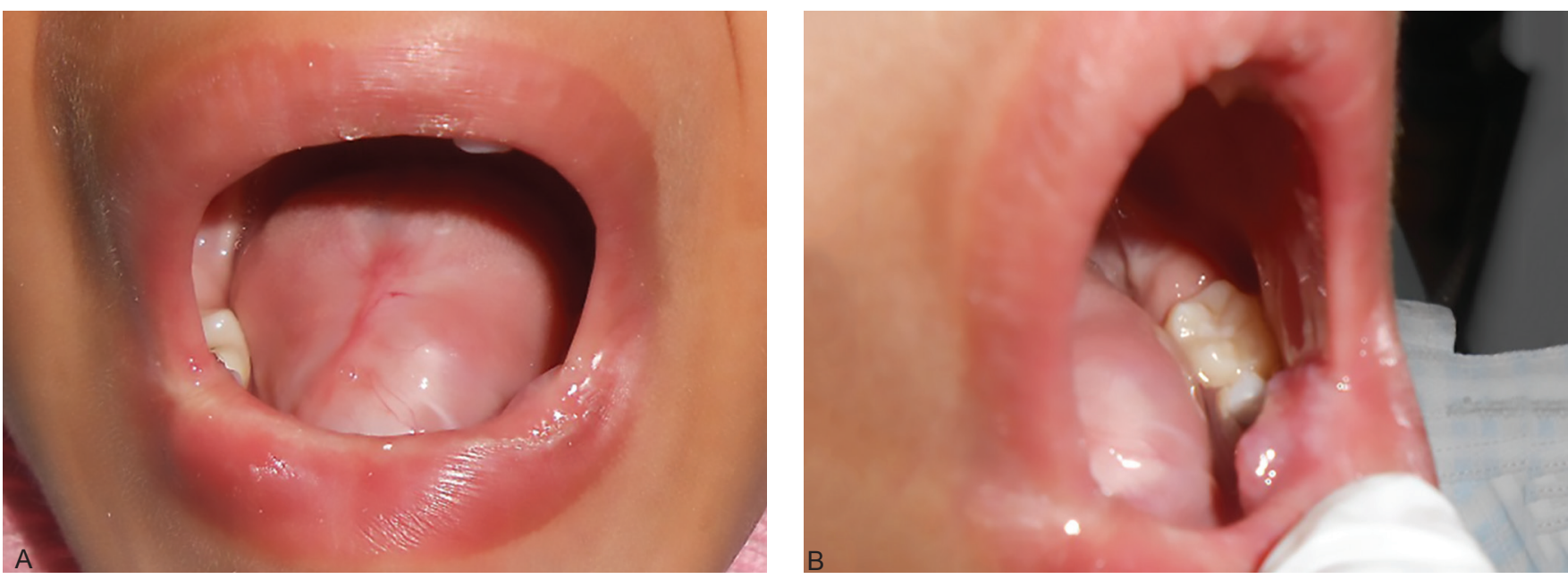

Figs 1A and B: (A) Post burns microstomia; (B) Scars at corner of the mouth

dentition. This case report presents the fabrication and effective use of extraoral commissure splint which helped in preventing lip adhesions in the post-surgical phase and achieving normal mouth opening for the child.

\section{METHODOLOGY}

An 8-year-old male child hailing from Nepal whose father was a daily wage worker was referred to the Department of Prosthodontics, AIIMS, New Delhi by the oral surgeon for fabrication of an oral screen appliance with extensions in commissure regions to keep lip tissues apart during healing. The patient was planned for excision of scar tissue on both sides of lips. Detailed case history was taken, and it revealed that the child had accidentally put some corrosive powder into the mouth around 6 months back which resulted in charring or burning of lips and later on scar formation on both lip corners. The scar caused a reduction in mouth opening which was found to be approximately $20 \mathrm{~mm}$ in the vertical direction at the time of presentation (Fig. 1A).

Intra-oral examination of the patient revealed that he had lip scars present on both corners of the lip and mild depapillation of the tongue was also seen (Fig. 1B). The patient presented with missing deciduous maxillary central incisors (51 and 61) but permanent central incisors were not visible clinically. All maxillary and mandibular deciduous second molars and mandibular deciduous first molars were grossly carious. Full mouth radiograph (OPG) was taken to ascertain the status of permanent teeth and to evaluate the extent of caries.

\section{Treatment Plan}

Involved three phases primarily:

- Prosthodontic phase: To fabricate the required postsurgical stent

- Surgical phase: For excision of scar tissue in the bilateral commissure regions to increase the mouth opening.
- Post-surgical evaluation by the prosthodontist for evaluation of appliance and pediatric dentist to treat carious lesions.

\section{Steps of Fabrication of Appliance}

Impressions of oral commissure were made before surgery. To make an impression of the commissures, cheek retractors (unilateral type) were used as impression trays (Fig. 2). Impressions were made for both the commissures separately in extended position using irreversible hydrocolloid to exert some horizontal and vertical pull in the commissure regions and to prevent lip adhesions post surgically. This was followed by pouring of impressions in type III stone and working models were obtained (Fig. 3A). Wax-up was done on both the models (Fig. 3B), and they were invested in type II plaster in a flask Model with wax up photo). After dewaxing and application of separating media (DPI Cold mold seal), self-cure acrylic resin (DPI, India) was mixed and packed into the molds and placed in a pressure pot to complete the polymerization. The appliance was carefully deflasked and finished and polished using the standard procedure. The appliance was tried

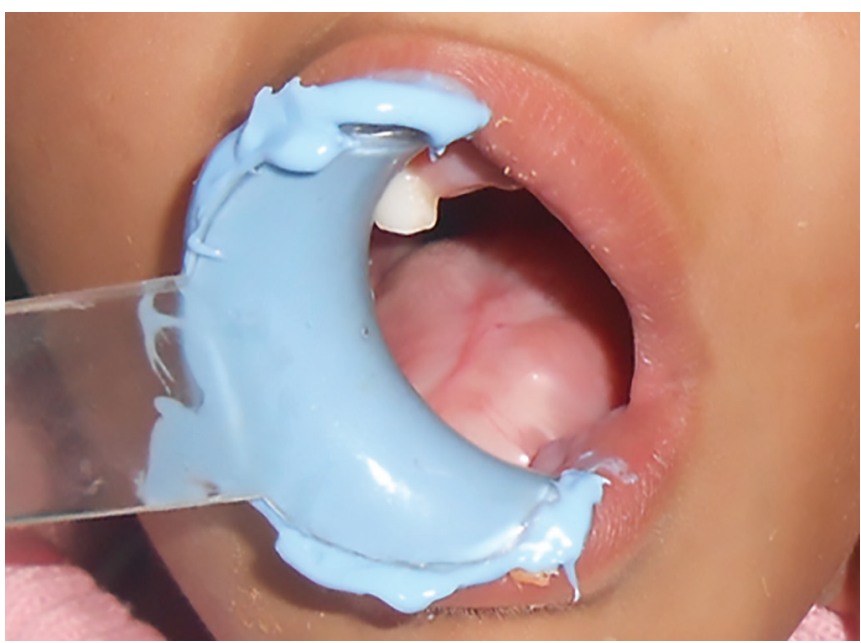

Fig. 2: Impression of commissures 

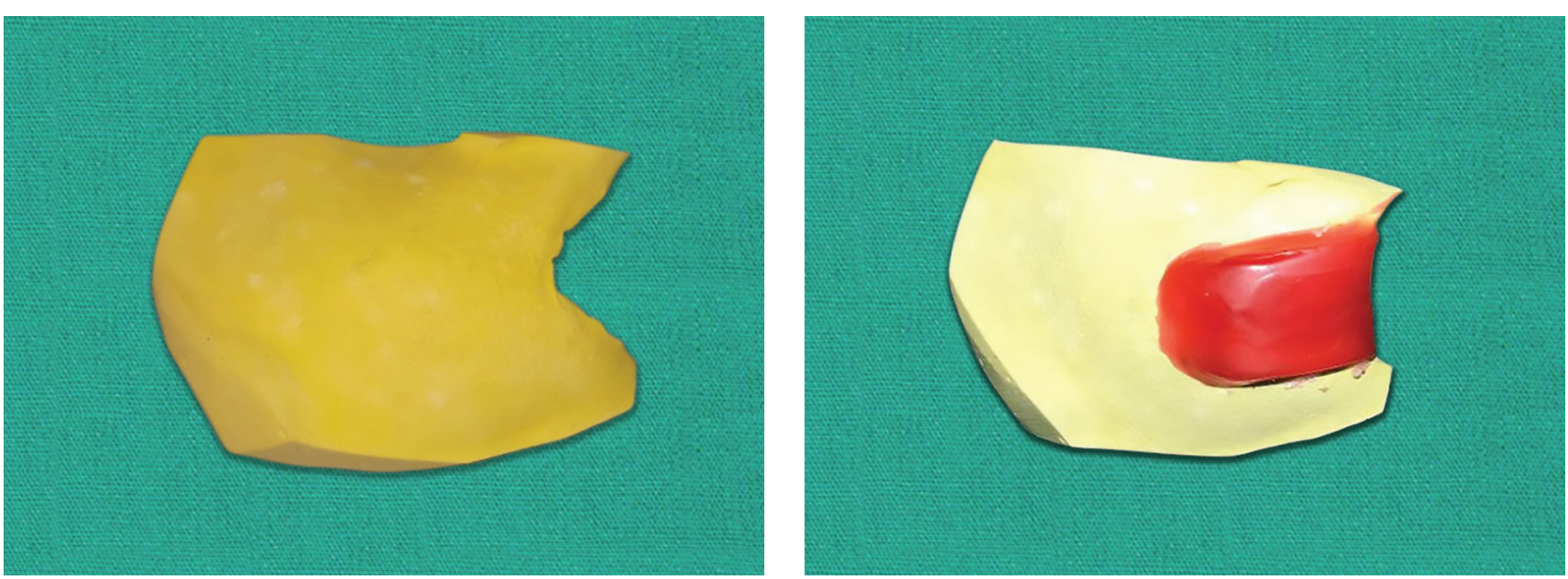

Figs 3A and B: (A) Cast obtained; (B) Wax up of appliance

on the patient's mouth, and necessary adjustments were done (Fig. 4A). The appliance was stabilized using extraoral elastic bandage taking anchorage from the head (Fig. 4B) and was delivered on the day of surgery after minor adjustments. The patient was instructed to wear the appliance for maximum time except during eating food and for cleaning. Regular follow-up visits were scheduled after 2 days, at one week, 6 months and one year later. The head elastic was changed at 6 months follow-up. Follow up at 1-year post-treatment showed increased mouth opening and complete tissue healing (Fig. 5).

\section{DISCUSSION AND SUMMARY}

Cooperation of child and parents is crucial for the success of such treatment along with the expertise of the treating dentist. A multidisciplinary treatment approach helped in overcoming various challenges in achieving the desired results.

The post-surgical removable stent was planned for this patient to keep lip tissues apart during the healing period to prevent epithelization as lip contracture may occur for

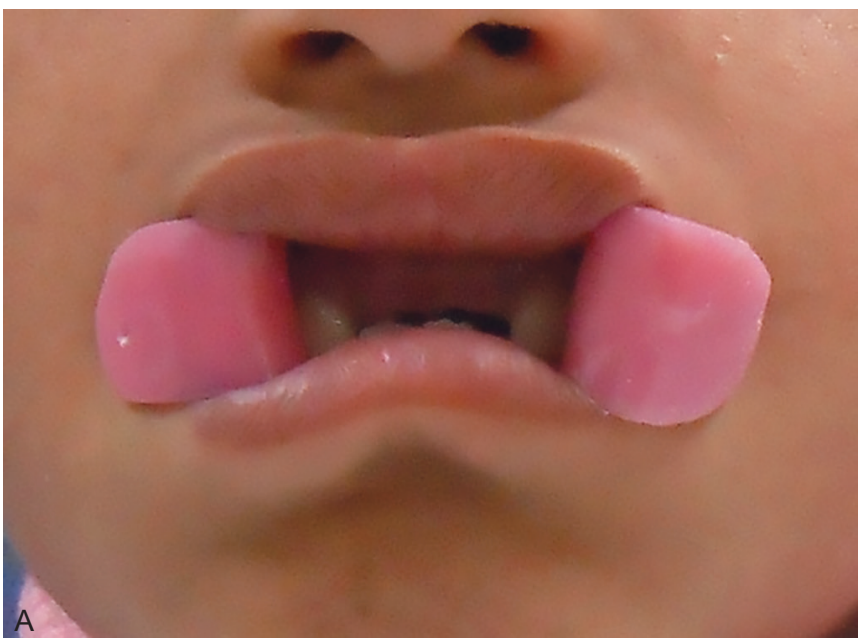

many months after the wound healing process is complete. Microstomia was the main challenge due to which making of intraoral maxillary or mandibular impression with one piece tray was not possible. Another limiting factor was the presence of partially erupted and carious teeth due to which obtaining intraoral retention for the appliance was also difficult. Intraoral extensions would have caused difficulty in the eruption of permanent teeth as the patient was about to enter mixed dentition stage with the scheduled eruption of permanent central incisors. So it was decided to take extraoral retention with the help of the elastic band. It maintained the definite pull and also reduced the risk of inadvertent swallowing of the appliance. So, an extraoral appliance was best suited for the patient.

Next challenge was to make the impression of commissure regions only as no specific trays are available for this purpose. So an innovative and simple method of making an impression with the help of easily available cheek retractors was used. This was an effective technique which can be used in future also.

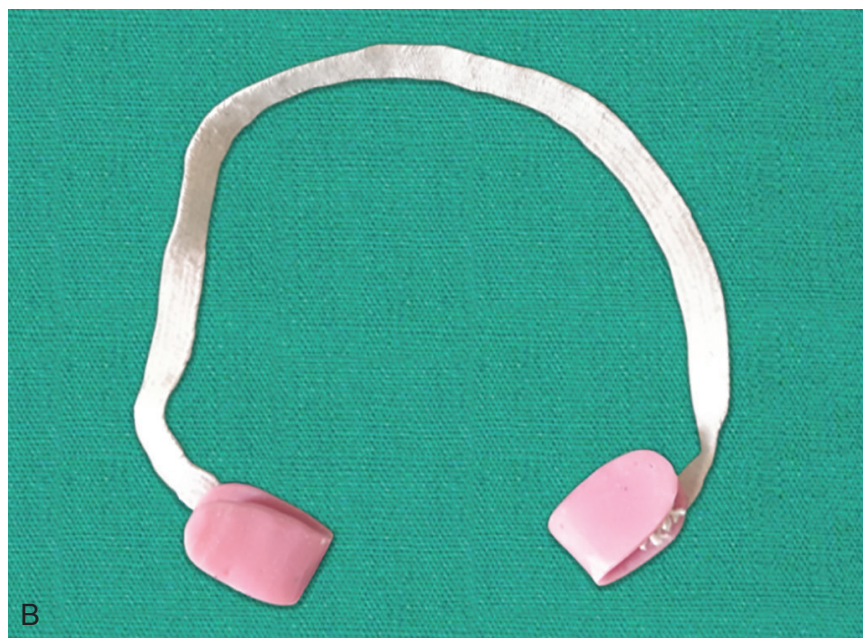

Figs 4A and B: (A) Appliance try in on patient's face; (B) Elastic band for extra oral anchorage 


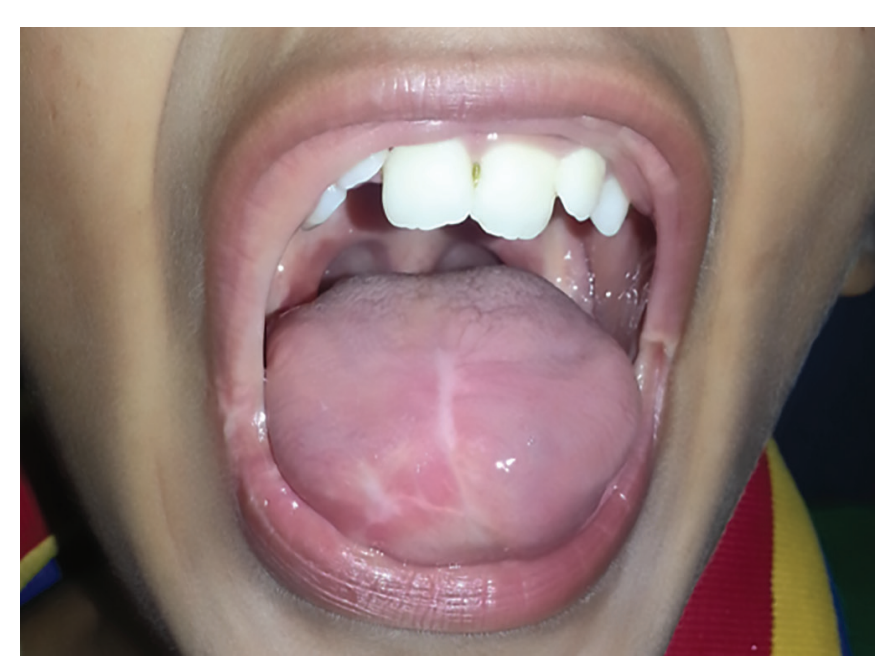

Fig. 5: Sufficient mouth opening at follow-up

\section{CLINICAL SIGNIFICANCE AND CONCLUSION}

The advantages of appliance given in our patient were: an innovative technique for the impression of commissures, no requirement of intraoral impressions or mouth preparation, easy fabrication, removable, inexpensive, indicated for children with partially erupted dentition, good patient compliance, achievement of both vertical and horizontal stretching of commissures symmetrically. The patient was referred to the pediatric dentist for follow up of intraoral treatment once he achieved adequate mouth opening.

\section{REFERENCES}

1. Bedard JF, Thongthammachat S, Toljanic JA. Adjunctive commissure splint therapy: A revised approach. J Prosthet Dent 2003;89:408-411.
2. Khan Z, Banis JC. Oral commissure expansion prosthesis. J Prosthet Dent 1992;67:383-385.

3. Wolfaardt JF, Levesque R. A technique for construction of dynamic oral commissure retractors. J Prosthet Dent 1990;64:195-197.

4. Czerepak CS. Oral splint therapy to manage electrical burns of the mouth in children. Clinics in plastic surgery. 1984 Oct;11(4):685-692.

5. Dahl E, Fogh-Andersen P. Electric burns of the mouth, longterm effects on the dentition: Surgical and orthodontic considerations. The European Journal of Orthodontics. 1980 Jan 1;2(4):207-217.

6. Nair CK, Sivagami G, Kunnekel AT, Naidu ME. Dynamic commissural splint. Indian J Dent Res 2008;19:165-168.

7. Carlow DL, Conine TA, Stevenson-Moore P. Static orthoses for the management of microstomia. J Rehabil Res Dev 1987;24: $35-42$.

8. LeCompte EJ, Goldman BM. Oral electric burns in children early treatment and appliance fabrication. Pediatric Dentistry 1982;4:333-337.

9. Taylor LB, Walker J. A review of selected microstomia prevention appliances. Pediatric Dentistry 1997;19:413-418.

10. Schneider PE. Infant commissural burn management with reverse pull headgear. Pediatric dentistry 1998;10: 34-38.

11. Naylor WP, Manor RC. Fabrication of a flexible prosthesis for the edentulous scleroderma patient with microstomia. J Prosthet Dent 1983;50:536-538.

12. Seals RR, Cain JR. Prosthetic treatment for chemical burns of the oral cavity. J Prosthet Dent 1985;53:688-691.

13. Clark WR, Mcdade GO. Microstomia in burn victims: A new appliance for prevention and treatment, and literature review. J Burn Care Rehab 1980;1:33-36.

14. Gorham JA. A mouth splint for burn microstomia. Am J Occup Ther 1977;31:105-106. 